\title{
VIBRATION ANALYSIS OF BEAMS SUBJECTED TO RANDOM EXCITATION BY THE DUAL CRITERION OF EQUIVALENT LINEARIZATION
}

\author{
Nguyen Nhu Hieu ${ }^{1, *}$, Nguyen Dong Anh ${ }^{1}$, Ninh Quang Hai ${ }^{2}$ \\ ${ }^{1}$ Institute of Mechanics, Vietnam Academy of Science and Technology, Hanoi, Vietnam \\ ${ }^{2}$ Hanoi Architectural University, Vietnam \\ *E-mail: nhuhieu1412@gmail.com \\ Received July 29, 2015
}

\begin{abstract}
In this paper responses of beams subjected to random loading are analyzed by the dual approach of the equivalent linearization method. The external random loading is assumed to be a space-wise and time-wise white noise in which the exact solutions of the modal equations can be found. A system of nonlinear algebraic equations for linearization coefficients of the modal linearized system is obtained in a closed form and is solved by the fixed-point iteration method. Results obtained from the proposed dual criterion are compared with the exact solution and those obtained from other approaches including energy method, and conventional linearization method. It is observed that the solution obtained by the dual criterion is in good agreement with the exact solution, especially, in the case of strong nonlinearity of beam.
\end{abstract}

Keywords: Random vibration, equivalent linearization, dual criterion, modal response, nonlinear beam.

\section{INTRODUCTION}

Over decades, the equivalent linearization (EQL) is one of the most extensively used methods in investigating mechanical systems. The earliest researches on the EQL method were carried out by Booton [1], Kazakov [2] and Caughey [3,4]. The fundamental idea of the method lies on replacing the original nonlinear system under a random external excitation by a linearized system under the same excitation in which linearization coefficients are found from a specified optimal criterion, for example, the mean-square error criterion [4], spectral criterion [5]. This method has developed and been applied successfully to find approximate responses of nonlinear system subjected random excitation. For discrete systems with single- and multi-degree-of-freedom, studies using the equivalent 
linearization can be found in some works [6-11], review articles [12-16], and in monographs by Crandall and Mark [17], Lin [18], Roberts and Spanos [19], Socha [5] with references therein. For continuous systems, the method of EQL is also applied [14,20-26]. In vibration analysis of beam structures under random excitations, the EQL method is studied by several authors. In Ref. [21], Herberts considered the effect of the membrane force on the stresses in a simply supported Bernoulli-Euler beam by the method of EQL. Seide [22] investigated nonlinear mean-square multimode responses of beams subjected to uniform pressure uncorrelated in time. Using EQL method, he obtained mean-square stresses and displacements of beams with arbitrary end conditions. Iwan and Whirley [23] developed a version of the EQL that can be applied to continuous systems under non-stationary random excitations. Their technique allows the replacement of the original nonlinear system with a time-varying linear continuous system. In [24,25], a new technique of equivalent linearization method is proposed based on the energy approach. Unlike the traditional replacement of EQL method [3,4], the energy method requires that the mean-square error between the potential energy of the original nonlinear system and that of corresponding linearized system must be minimum. In [26], Anh et al. extended the approach of regulated equivalent linearization (RGEL) in studying single-degree-of-freedom system to random vibration analysis of beams under random loadings. The effective of the RGEL method is recorded by its excellent performance in calculating approximate modal responses of the beam.

Recently, Anh et al. [27] have proposed a dual criterion of the EQL method for nonlinear single-degree-of-freedom systems under random excitations. The authors showed that the accuracy of the mean-square response obtained by the dual criterion is significantly improved when the nonlinearity is increasing. This dual approach is then extended to cases of multi-degree-of-freedom systems [28].

Naturally, the dual approach may be extended to random vibration analysis of many continuous systems. In this research, a version of dual criterion of the EQL method is developed for analyzing modal responses of beams subjected to random loading. A nonlinear algebraic system of linearization coefficients obtained in a closed form is solved by an iteration method. To elucidate the dual approach, the obtained results are compared with those of the conventional linearization and energy methods.

\section{THE GOVERNING EQUATION OF BEAM}

Consider the governing equation of a beam on elastic foundation, restrained at its ends and subjected to a space-wise distributed time-dependent loading $p(x, t)[24,25]$

$$
E I \frac{\partial^{4} w}{\partial x^{4}}-N \frac{\partial^{2} w}{\partial x^{2}}+\mu A \frac{\partial^{2} w}{\partial t^{2}}+\beta \frac{\partial w}{\partial t}+K_{f} w=p(x, t),
$$

where the axial force $N$ is given by

$$
N=\frac{E A}{2 L} \int_{0}^{L}\left(\frac{\partial w}{\partial x}\right)^{2} d x .
$$


Here, $A$ and $I$ are the area and inertia moment of the cross-section, respectively; $E$ is the elastic modulus, $\mu$ the mass density, $\beta$ the viscous damping coefficient, $L$ the length of the beam, $K_{f}$ the stiffness of the elastic foundation; $w(x, t)$ is the deflection of the beam. In this paper, a space-wise and time-wise white noise loading $p(x, t)$ is considered.

In order to solve (1) one expands $p(x, t)$ in series [24]

$$
p(x, t)=\sum_{n=1}^{\infty} q_{n}(t) \phi_{n}(x),
$$

where $q_{n}(t)(n=1,2, \ldots)$ are zero-mean Gaussian white noise stationary random processes with corresponding correlations

$$
E\left[q_{m}(t) q_{n}(t+\tau)\right]=2 \pi S_{m} \delta_{m n} \delta(\tau),(m, n=1,2, \ldots)
$$

in which $\delta_{m n}$ is the Kronecker-delta notation, $\delta(\tau)$ is the Dirac-delta; the quantities $S_{n}$ $(n=1,2, \ldots)$ are constant spectral density values of random processes $q_{n}(t)$. The functions $\phi_{n}(x)(n=1,2, \ldots)$ in the series (3) are modal shapes satisfying the following relationships

$$
\begin{gathered}
\frac{d^{4} \phi_{n}}{d x^{4}}=\frac{\mu A}{E I} \omega_{n}^{2} \phi_{n}, \\
\int_{0}^{1} \phi_{m} \phi_{n} d \xi=\delta_{m n}, \quad \xi=\frac{x}{L}
\end{gathered}
$$

where $\omega_{n}(n=1,2, \ldots)$ are natural frequencies associated with free vibration of the system (1) without the axial force, viscous damping, elastic foundation and external excitation for a simply supported boundary condition

$$
\omega_{n}^{2}=\frac{n^{4} E I \pi^{4}}{\mu A L^{4}} .
$$

Let the deflection function $w(x, t)$ of the beam be expended in terms of an appropriate orthogonal set of modal shapes $\phi_{n}(x)$ as follows

$$
w(x, t)=\sum_{n=1}^{\infty} w_{n}(t) \phi_{n}(x)
$$

where $w_{n}(t)$ is the modal contribution corresponding to $n^{t h}$-mode. Substituting Eq. (8) into the expression of the axial force $N$ in Eq. (2) yields

$$
N=\frac{E A}{2 L^{2}} \sum_{n=1}^{\infty} \sum_{m=1}^{\infty} K_{n m} w_{n} w_{m}
$$

where it is denoted

$$
K_{n m}=\int_{0}^{1} \frac{d \phi_{n}}{d \xi} \frac{d \phi_{m}}{d \xi} d \xi=K_{m n}
$$


In view of Eqs. (5) and (6), the governing equation (1) takes the form

$$
\sum_{n=1}^{\infty}\left(\mu A \ddot{w}_{n}+\beta \dot{w}_{n}+K_{f} w_{n}+\mu A \omega_{n}^{2} w_{n}\right) \phi_{n}-\frac{E A}{2 L^{2}} \sum_{n=1}^{\infty} \sum_{i=1}^{\infty} \sum_{j=1}^{\infty} K_{i j} w_{i} w_{j} w_{n} \frac{d^{2} \phi_{n}}{d x^{2}}=p(x, t) .
$$

Multiplication of Eq. (11) by $\phi_{m}$, integration over the length of the beam, and then use of orthogonality conditions given in Eq. (6) yield a set of coupled nonlinear differential equations for modal amplitudes $w_{m}(t)$

$$
\ddot{w}_{m}+\frac{\beta}{\mu A} \dot{w}_{m}+\omega_{m}^{2} w_{m}+\frac{K_{f}}{\mu A} w_{m}+\frac{E}{2 \mu L^{4}} \sum_{n=1}^{\infty} \sum_{i=1}^{\infty} \sum_{j=1}^{\infty} K_{i j} K_{n m} w_{i} w_{j} w_{n}=b_{m}(t),
$$

where the random function $b_{m}(t)$ is given by

$$
b_{m}(t)=\frac{1}{\mu A L} \int_{0}^{L} p(x, t) \phi_{m}(x) d x .
$$

Further in the expansion (8), it is assumed that only the first $M$ modes of the beam significantly contribute to formulate responses. Crandall and Yildiz [29] shown that if the infinite series that are representing quantities such as displacement, mean-square stresses, etc., converge, then the results can be made as accurate as desired by taking sufficiently large $M$. For this reason, Eq. (12) can be taken by the following finite form with the first $M$ modes

$$
\ddot{w}_{m}+\frac{\beta}{\mu A} \dot{w}_{m}+\omega_{m}^{2} w_{m}+\frac{K_{f}}{\mu A} w_{m}+G_{m}\left(w_{1}, w_{2}, \ldots, w_{M}\right)=b_{m}(t),
$$

in which nonlinear components $G_{m}(m=1,2, \ldots, M)$ are functions of $M$ variables $w_{1}, \ldots, w_{M}$

$$
G_{m}=G_{m}\left(w_{1}, w_{2}, \ldots, w_{M}\right)=\frac{E}{2 \mu L^{4}} \sum_{n=1}^{M} \sum_{i=1}^{M} \sum_{j=1}^{M} K_{i j} K_{n m} w_{i} w_{j} w_{n} .
$$

Our objective is to find approximate mean-square modal responses of the beam from the modal system (14). In the next section, the dual criterion of stochastic linearization method will be applied to this nonlinear system.

\section{A DUAL CRITERION FOR MODAL EQUATIONS OF BEAM}

The dual criterion of stochastic linearization method appears from an idea that the original nonlinear system can be replaced by an equivalent linearization system, and then this equivalent system is replaced by another nonlinear system that belongs to the same class of the original nonlinear system. Some obtained results using the dual criterion are presented in works of Anh et al. [27,28] for single- and multi-degree-of-freedom systems subjected to random excitations. Naturally, the dual criterion of stochastic linearization needs to be developed in investigating continuous systems under random excitation. For 
the governing equation of the beam in the modal form (14), one can make a linearization version as follows

$$
\ddot{w}_{m}+\frac{\beta}{\mu A} \dot{w}_{m}+\omega_{m}^{2} w_{m}+\frac{K_{f}}{\mu A} w_{m}+\omega_{m}^{2} k_{e q, m} w_{m}=b_{m}(t),
$$

where $k_{e q, m}(m=1,2, \ldots, M)$ are non-dimensional linearization coefficients determined from a specified criterion of stochastic linearization. We here utilize the dual criterion $[27,28]$ for determining coefficients $k_{e q, m}(m=1,2, \ldots, M)$. In the first step, the original nonlinear term $G_{m}$ is replaced by a linearized one $\omega_{m}^{2} k_{e q, m} w_{m}$, and then the linear term $\omega_{m}^{2} k_{e q, m} w_{m}$ is replaced by another nonlinear quantity, $\lambda_{m} G_{m}$, that can be considered as a term belonging to the same class of the original function $G_{m}$, where the coefficients $k_{\text {eq, }}$ and $\lambda_{m}$ are determined from the following proposed criterion for beam vibration

$$
e_{1}=E\left[\left(G_{m}-\omega_{m}^{2} k_{e q, m} w_{m}\right)^{2}\right]+\rho E\left[\left(\omega_{m}^{2} k_{e q, m} w_{m}-\lambda_{m} G_{m}\right)^{2}\right] \rightarrow \min _{k_{e q, m}, \lambda_{m}},
$$

with the detuning parameter $\rho$ taking two values 0 or 1 . When the parameter $\rho$ is equal to zero, the criterion (17) becomes the conventional mean-square error criterion which can be found in the literature. On the other hand, as the parameter $\rho$ is taken to be 1 , the criterion (19) is so-called dual one. In this criterion, the first expectation can be understood as a component of the conventional replacement, whereas the second one describes a dual replacement of the linearization problem. Similar to the conventional linearization (see [6]), the criterion (17) leads to that partial derivatives of the expression $e_{1}$ with respect to variables $k_{e q, m}$ and $\lambda_{m}$ are equal to zero

$$
\frac{\partial e_{1}}{\partial k_{e q, m}}=0, \quad \frac{\partial e_{1}}{\partial \lambda_{m}}=0,(m=1,2, \ldots, M) .
$$

The system (18) yields a set of algebraic equations of variables $k_{e q, m}$ and $\lambda_{m}$, $(m=1,2, \ldots, M)$ as follows

$$
\begin{aligned}
& \left((1+\rho) \omega_{m}^{2} E\left[w_{m}^{2}\right]\right) k_{e q, m}=\left(E\left[w_{m} G_{m}\right]\right)\left(1+\rho \lambda_{m}\right), \\
& \lambda_{m}=\left(\omega_{m}^{2} \frac{E\left[w_{m} G_{m}\right]}{E\left[G_{m}^{2}\right]}\right) k_{\text {eq, } m .}
\end{aligned}
$$

Solving the system (19) for unknowns $k_{e q, m}$ and $\lambda_{m}$, we arrive at

$$
\begin{gathered}
k_{e q, m}=\frac{1}{\omega_{m}^{2}} \frac{1}{1+\rho-\rho \eta_{m}} \frac{E\left[w_{m} G_{m}\right]}{E\left[w_{m}^{2}\right]}, \\
\lambda_{m}=\frac{\eta_{m}}{1+\rho-\rho \eta_{m}},
\end{gathered}
$$

where

$$
\eta_{m}=\frac{\left(E\left[w_{m} G_{m}\right]\right)^{2}}{E\left[w_{m}^{2}\right] E\left[G_{m}^{2}\right]} .
$$

It is observed that, using the dual criterion (17), the original nonlinear Eq. (14) of modes of beam vibration is replaced by its linearization version (16), in which linearization coefficients $k_{e q, m}(m=1,2, \ldots, M)$ are determined from expressions (20)-(22). In the 
framework of this article, the dual criterion (17) is elucidated for random vibrations of a simply supported beam under a space-wise and time-wise white noise loading.

\section{RESPONSES OF A SIMPLY SUPPORTED BEAM AT BOTH ENDS}

\subsection{Equivalent linearization coefficients}

For a simply supported beam, one has the following expression for the modal shape $\phi_{m}[25]$

$$
\phi_{m}(\xi)=\sqrt{2} \sin (m \pi \xi)
$$

Using the expressions (10) and (23), one obtain

$$
K_{n m}=K_{m n}=\int_{0}^{1} \frac{d \phi_{n}}{d \xi} \frac{d \phi_{m}}{d \xi} d \xi= \begin{cases}\pi^{2} m^{2} & \text { if } m=n \\ 0 & \text { if } m \neq n\end{cases}
$$

Eq. (14) becomes

$$
\ddot{w}_{m}+\frac{\beta}{\mu A} \dot{w}_{m}+\omega_{m}^{2}\left(1+\frac{\alpha}{m^{4}}\right) w_{m}+\frac{\omega_{m}^{2}}{2 R^{2} m^{2}} \sum_{n=1}^{M} n^{2} w_{n}^{2} w_{m}=b_{m}(t),
$$

where

$$
\omega_{m}^{2}=\omega_{0}^{2} m^{4}, \quad \omega_{0}^{2}=\frac{E I \pi^{4}}{\mu A L^{4}}, \quad \alpha=\frac{K_{f}}{\mu A \omega_{0}^{2}}, \quad R=\sqrt{\frac{I}{A}} .
$$

The nonlinear functions $G_{m}(m=1,2, \ldots, M)$ in Eq. (25) take the form

$$
G_{m}=\frac{\omega_{m}^{2}}{2 R^{2} m^{2}} \sum_{n=1}^{M} n^{2} w_{n}^{2} w_{m} .
$$

Substituting expressions $G_{m}(m=1,2, \ldots, M)$ from Eq. (27) into Eq. (20) yields

$$
k_{e q, m}=\frac{1}{2 R^{2} m^{2}} \frac{1}{1+\rho-\rho \eta_{m}} \frac{\sum_{n=1}^{M} n^{2} E\left[w_{n}^{2} w_{m}^{2}\right]}{E\left[w_{m}^{2}\right]},
$$

where

$$
\eta_{m}=\frac{\sum_{i=1}^{M} \sum_{j=1}^{M} i^{2} j^{2} E\left[w_{i}^{2} w_{m}^{2}\right] E\left[w_{j}^{2} w_{m}^{2}\right]}{E\left[w_{m}^{2}\right] \sum_{i=1}^{M} \sum_{j=1}^{M} i^{2} j^{2} E\left[w_{i}^{2} w_{j}^{2} w_{m}^{2}\right]} .
$$

It is noted that, to calculate higher-order moments in Eqs. (28) and (29), we employ the following generalized formula expressed in terms of second-order moments of Gaussian random processes with zero-mean [30]

$$
E\left[z_{1} z_{2} \ldots z_{2 m}\right]=\sum_{\text {all independent pairs }}\left(\prod_{j \neq k} E\left[z_{j} z_{k}\right]\right),
$$


where the number of independent pairs is equal to $(2 m) ! /\left(2^{m} m !\right)$. Particularly, in view of the present dual method for beam vibration, the following higher-order moment terms will appear

$$
\begin{aligned}
& E\left[w_{m}^{2} w_{n}^{2}\right]=E\left[w_{m}^{2}\right] E\left[w_{n}^{2}\right]+2\left(E\left[w_{m} w_{n}\right]\right)^{2}=y_{m m} y_{n n}+2 y_{m n}^{2}, \\
& E\left[w_{j}^{2} w_{j}^{2} w_{m}^{2}\right]= E\left[w_{i}^{2}\right] E\left[w_{j}^{2}\right] E\left[w_{m}^{2}\right]+2\left(E\left[w_{i} w_{j}\right]\right)^{2} E\left[w_{m}^{2}\right] \\
&+2\left(E\left[w_{j} w_{m}\right]\right)^{2} E\left[w_{i}^{2}\right]+2\left(E\left[w_{i} w_{m}\right]\right)^{2} E\left[w_{j}^{2}\right] \\
&+8 E\left[w_{i} w_{j}\right] E\left[w_{j} w_{m}\right] E\left[w_{i} w_{m}\right] \\
&= y_{i i} y_{j j} y_{m m}+2 y_{i j}^{2} y_{m m}+2 y_{j m}^{2} y_{i i}+2 y_{i m}^{2} y_{j j}+8 y_{i j} y_{j m} y_{i m},
\end{aligned}
$$

where the notation $y_{m n}$ for the second moment of $w_{m}$ is introduced

$$
y_{m n}=y_{n m}=E\left[w_{m} w_{n}\right] \text {. }
$$

Substituting expressions (31) in Eq. (28), we arrive at

$$
k_{e q, m}=\frac{1}{2 R^{2} m^{2}} \frac{1}{1+\rho-\rho \eta_{m}} \sum_{n=1}^{M} n^{2} \frac{y_{n n} y_{m m}+2 y_{m n}^{2}}{y_{m m}},
$$

where

$$
\eta_{m}=\frac{\sum_{i=1}^{M} \sum_{j=1}^{M} i^{2} j^{2}\left(y_{i i} y_{m m}+2 y_{i m}^{2}\right)\left(y_{j j} y_{m m}+2 y_{j m}^{2}\right)}{y_{m m} \sum_{i=1}^{M} \sum_{j=1}^{M} i^{2} j^{2}\left(y_{i i} y_{j j} y_{m m}+2 y_{i j}^{2} y_{m m}+2 y_{j m}^{2} y_{i i}+2 y_{i m}^{2} y_{j j}+8 y_{i j} y_{j m} y_{i m}\right)} .
$$

For purpose of comparing the present dual criterion with other methods, in this paper, we also present two known results of the conventional linearization [21,22], and energy method [24,25]. From the linearized system (16), the following expressions of the equivalent linearization coefficients $k_{e q, m}(m=1,2, \ldots, M)$ are obtained using the conventional linearization method

$$
k_{e q, m_{-} \text {conventional }}=\frac{1}{2 R^{2} m^{2}} \sum_{n=1}^{M} n^{2} \frac{y_{n n} y_{m m}+2 y_{n m}^{2}}{y_{m m}} .
$$

It is observed that the expression (35) is also obtained from (33) by taking the detuning parameter $\rho$ be zero. The linearization method based on energy criterion gives the equivalent coefficients $k_{e q, m}(m=1,2, \ldots, M)$ obtained from the following system [24,25]

$$
\begin{aligned}
\left\{1+\alpha+k_{1, e q}, 2^{4}\right. & \left.\left(1+\frac{\alpha}{2^{4}}+k_{2, e q}\right), \ldots, M^{4}\left(1+\frac{\alpha}{M^{4}}+k_{M, e q}\right)\right\}^{T} \\
& =\frac{2}{\omega_{0}^{2}} \mathbf{A}^{-1}\left\{E\left[w_{1}^{2} U\right] \quad E\left[w_{2}^{2} U\right] \ldots E\left[w_{M}^{2} U\right]\right\}^{T},
\end{aligned}
$$


where the matrix $\mathbf{A}$ and potential energy $U$ of the system are determined as follows

$$
\begin{aligned}
& \mathbf{A}=\left[\begin{array}{llll}
E\left[w_{1}^{2} w_{1}^{2}\right] & E\left[w_{1}^{2} w_{2}^{2}\right] & \ldots & E\left[w_{1}^{2} w_{M}^{2}\right] \\
E\left[w_{2}^{2} w_{1}^{2}\right] & E\left[w_{2}^{2} w_{2}^{2}\right] & \ldots & E\left[w_{2}^{2} w_{M}^{2}\right] \\
\ldots & \ldots & \ldots & \ldots \\
E\left[w_{M}^{2} w_{1}^{2}\right] & E\left[w_{M}^{2} w_{2}^{2}\right] & \ldots & E\left[w_{M}^{2} w_{M}^{2}\right]
\end{array}\right], \\
& U=\frac{\omega_{0}^{2}}{2}\left[\sum_{m=1}^{M}\left(\alpha+m^{4}\right) w_{m}^{2}+\frac{1}{4 R^{2}}\left(\sum_{m=1}^{M} m^{2} w_{m}^{2}\right)^{2}\right] .
\end{aligned}
$$

To get a closed form of the equivalent linearization coefficients $k_{e q, m}(m=1,2, \ldots, M)$ in Eq. (33), we utilize responses of the linearized (16) via spectral density of the external excitations.

\subsection{Responses of the linearized system}

For the linearized system (16) under the random excitation $b$, one can obtain secondorder moments $E\left[w_{m} w_{n}\right]$ of the responses $w_{m}$ as follows (see [19] for details)

$$
E\left[w_{m} w_{n}\right]=\int_{-\infty}^{\infty} H_{m}(-\omega) B_{m n}(\omega) H_{n}(\omega) d \omega,
$$

where

$$
B_{m n}(\omega)=\frac{S_{m} \delta_{m n}}{(\mu A)^{2}}
$$

and the frequency-response function $H_{m}(\omega)$ is given by

$$
H_{m}(\omega)=\frac{1}{\left(1+\frac{\alpha}{m^{4}}+k_{e q, m}\right) \omega_{m}^{2}-\omega^{2}+i \frac{\beta}{\mu A} \omega} .
$$

Because $B_{m n}(\omega)$ given by (40) are constants, moments $E\left[w_{m} w_{n}\right]$ can be rewritten as

$$
E\left[w_{m} w_{n}\right]=B_{m n} \int_{-\infty}^{\infty} H_{m}(-\omega) H_{n}(\omega) d \omega .
$$

Introducing (41) into the right-hand side of the expression (42) and employing residual theorem in theory of complex variable functions, we get

$$
\begin{array}{r}
y_{m n}=E\left[w_{m} w_{n}\right]=\frac{4 \pi \beta B_{m n}}{\mu A}\left\{\left[\left(1+\frac{\alpha}{m^{4}}+k_{e q, m}\right) \omega_{m}^{2}-\left(1+\frac{\alpha}{n^{4}}+k_{e q, n}\right) \omega_{n}^{2}\right]^{2}\right. \\
\left.+2\left(\frac{\beta}{\mu A}\right)^{2}\left[\left(1+\frac{\alpha}{m^{4}}+k_{e q, m}\right) \omega_{m}^{2}+\left(1+\frac{\alpha}{n^{4}}+k_{e q, n}\right) \omega_{n}^{2}\right]\right\}^{-1} .
\end{array}
$$

It is seen that a closed system of nonlinear algebraic equations for unknowns $k_{\text {eq, } m}$ ( $m=1,2, \ldots, M)$ is obtained by substituting (43) in to the right hand side of Eq. (33). As noted before, because the contribution of the first modes of the system is significant, we here restrict our calculations for modal responses of the beam in two cases: single-, and 
two-mode using three approaches: the conventional linearization, energy method, and present dual criterion.

\section{NUMERICAL RESULTS AND DISCUSSIONS}

It is seen that, in Eq. (25) for vibrational modes, as $R$ tends to infinity, the effect of nonlinear terms $G_{m}(m=1,2, \ldots, M)$ disappear. Therefore, one can view the magnitude of the quantity $1 / R$ as the parameter related to the magnitude of nonlinearity of the original nonlinear system (25). Assume that spectral densities $(m=1,2, \ldots, M)$ of the stochastic processes $q_{m}(t)$ have the same value, i.e. $S_{1}=S_{2}=\ldots=S_{M}=S_{0}$. For this assumption, from the Fokker-Planck equation corresponding to the system (25), the exact expression of the probability density function can be obtained [24]

$$
\begin{aligned}
P\left(w_{1}, w_{2}, \ldots, w_{M}\right) & =\frac{1}{C} \exp \left\{-\frac{\beta \mu A \omega_{0}^{2}}{2 \pi S_{0}}\left[\sum_{m=1}^{M}\left(\alpha+m^{4}\right) w_{m}^{2}+\frac{1}{4 R^{2}}\left(\sum_{m=1}^{M} m^{2} w_{m}^{2}\right)^{2}\right]\right\} \\
& =\frac{1}{C} \exp \left\{-\frac{\beta \mu A}{\pi S_{0}} U\left(w_{1}, w_{2}, \ldots, w_{M}\right)\right\},
\end{aligned}
$$

where $U=U\left(w_{1}, w_{2}, \ldots, w_{M}\right)$ is the potential energy of the system (25) given by (38), and $C$ is the normalization constant



The exact modal mean-square response of Eq. (25) is evaluated by the following multiple integral with $M$-fold

$$
E\left[w_{m}^{2}\right]_{\text {exact }}=\underbrace{\int_{-\infty}}_{\underbrace{-}_{-\infty} \int_{-\infty}^{\infty} \ldots \int_{-\infty}^{\infty} w_{m}^{2} \exp }\left\{-\frac{\beta \mu A \omega_{0}^{2}}{2 \pi S_{0}}\left[\sum_{m=1}^{M}\left(\alpha+m^{4}\right) w_{m}^{2}+\frac{1}{4 R^{2}}\left(\sum_{m=1}^{M} m^{2} w_{m}^{2}\right)^{2}\right]\right\} d w_{1} \ldots d w_{M} .
$$

In general, the multiple integral (46) must be calculated using a numerical method. In the following computation, we use the exact solution (46) in the case of single-mode $(M=1)$ and of two-mode $(M=2)$ to elucidate the accuracy of the proposed dual criterion method (33), and other methods for comparison purpose.

\subsection{The case of single-mode}

For the single-mode, $M=1$, the governing equation of the simply supported beam (25) takes the form

$$
\ddot{w}_{1}+\frac{\beta}{\mu A} \dot{w}_{1}+\omega_{0}^{2}(1+\alpha) w_{1}+\frac{\omega_{0}^{2}}{2 R^{2}} w_{1}^{3}=\frac{1}{\mu A} q_{1}(t) .
$$


This is the well-known Duffing oscillator subjected to random excitation $[4,5]$. From Eq. (46), one can get an exact solution of mean-square response of $w_{1}$ in Eq. (47) as follows (see also $[22,26]$ )

$$
\begin{aligned}
E\left[w_{1}^{2}\right]_{\text {exact }, 1} & =\frac{\int_{-\infty}^{\infty} w_{1}^{2} \exp \left\{-\frac{\beta \mu A}{\pi S_{0}}\left(\frac{1}{2} \omega_{0}^{2}(1+\alpha) w_{1}^{2}+\frac{\omega_{0}^{2}}{8 R^{2}} w_{1}^{4}\right)\right\} d w_{1}}{\int_{-\infty}^{\infty} \exp \left\{-\frac{\beta \mu A}{\pi S_{0}}\left(\frac{1}{2} \omega_{0}^{2}(1+\alpha) w_{1}^{2}+\frac{\omega_{0}^{2}}{8 R^{2}} w_{1}^{4}\right)\right\} d w_{1}} \\
& =(1+\alpha) R^{2}\left[K_{3 / 4}\left(\frac{(1+\alpha)^{2}}{4} \frac{R^{2}}{R_{01}^{2}}\right)-K_{1 / 4}\left(\frac{(1+\alpha)^{2}}{4} \frac{R^{2}}{R_{01}^{2}}\right)\right] / K_{1 / 4}\left(\frac{(1+\alpha)^{2}}{4} \frac{R^{2}}{R_{01}^{2}}\right)
\end{aligned}
$$

where $K_{v}(y)$ is the modified Bessel function of the second kind of order $v$ of the variable $y$, and the quantity $R_{01}$ is given by

$$
R_{01}=\sqrt{\frac{\pi S_{0}}{\beta(\mu A) \omega_{0}^{2}}} .
$$

Using Eqs. (43) and (28) for $M=1$, we get the following single-mode approximate mean-square response $E\left[w_{1}^{2}\right]$ depending upon the nonlinear parameter $1 / R$ in the following form

$$
E\left[w_{1}^{2}\right] \text { dual } 1=\frac{2 R_{01}^{2}}{1+\alpha+\sqrt{(1+\alpha)^{2}+\frac{30}{7} \frac{R_{01}^{2}}{R^{2}}}} .
$$

Similarly, approximate mean-square responses of $w_{1}$ corresponding to the conventional linearization (35) and energy method (36) in the case of single-mode are obtained, respectively,

$$
\begin{aligned}
& E\left[w_{1}^{2}\right]_{\text {conventional }, 1}=\frac{2 R_{01}^{2}}{1+\alpha+\sqrt{(1+\alpha)^{2}+\frac{6 R_{01}^{2}}{R^{2}}}}, \\
& E\left[w_{1}^{2}\right]_{\text {energy }, 1}=\frac{2 R_{01}^{2}}{1+\alpha+\sqrt{(1+\alpha)^{2}+\frac{5 R_{01}^{2}}{R^{2}}}} .
\end{aligned}
$$

Numerical results for the first mode of beam vibration in the case of single-mode using four methods, including the exact solution (48), conventional linearization (51), energy method (52), and dual criterion method (50) are illustrated in Tabs. 1 and 2. The system parameters are $\omega_{0}=1, \beta=0.1, \mu A=1$. Tab. 1 shows a comparison of relative errors between results obtained from approximate and exact solution methods. The stiffness parameter $\alpha$ of the system is fixed at 1 , whereas the nonlinearity parameter $1 / R$ varies from 0.01 to 10.0. It is seen that, for small values of $1 / R$, for example $1 / R=0.01,0.02,0.05$, the conventional linearization yields quite small errors, about $0.05 \%$, whereas errors of the energy and dual criterion methods are larger. In the range $[1,10]$ of $1 / R$, the error of conventional linearization becomes larger $10 \%$ while that of the energy method and 
Table 1. Mean-square response of $w_{1}$ of the simply supported beam in case of single-mode with $\omega_{0}=1, \alpha=1, \beta=0.1, \mu A=1, S_{0}=1$ and various values of $1 / R$ (CL: Conventional

Linearization; EM: Energy Method; DM: Dual Criterion Method)

\begin{tabular}{|c|c|c|c|c|c|c|c|}
\hline $1 / R$ & $E\left[w_{1}^{2}\right]_{\text {exact, } 1}$ & $E\left[w_{1}^{2}\right]_{\mathrm{CL}, 1}$ & Error $(\%)$ & $E\left[w_{1}^{2}\right]_{\mathrm{EM}, 1}$ & Error $(\%)$ & $E\left[w_{1}^{2}\right]_{\mathrm{DM}, 1}$ & Error (\%) \\
\hline 0.01 & 15.6895 & 15.6895 & 0.0001 & 15.6926 & 0.0195 & 15.6948 & 0.0335 \\
\hline 0.02 & 15.6349 & 15.6346 & 0.0014 & 15.6468 & 0.0761 & 15.6554 & 0.1317 \\
\hline 0.05 & 15.2778 & 15.2707 & 0.0466 & 15.3403 & 0.4086 & 15.3907 & 0.7388 \\
\hline 0.10 & 14.2613 & 14.1964 & 0.4549 & 14.4101 & 1.0437 & 14.5706 & 2.1690 \\
\hline 0.20 & 11.9200 & 11.6419 & 2.3324 & 12.0674 & 1.2370 & 12.4086 & 4.0991 \\
\hline 0.50 & 7.3952 & 6.8668 & 7.1448 & 7.3248 & 0.9518 & 7.7220 & 4.4191 \\
\hline 1.00 & 4.4131 & 3.9581 & 10.3102 & 4.2767 & 3.0909 & 4.5614 & 3.3618 \\
\hline 2.00 & 2.4261 & 2.1276 & 12.3034 & 2.3146 & 4.5968 & 2.4842 & 2.3928 \\
\hline 5.00 & 1.0294 & 0.8890 & 13.6400 & 0.9712 & 5.6599 & 1.0463 & 1.6388 \\
\hline 10.00 & 0.5251 & 0.4510 & 14.1093 & 0.4934 & 6.0421 & 0.5322 & 1.3564 \\
\hline
\end{tabular}

Table 2. Mean-square response of $w_{1}$ of the simply supported beam in case of single-mode with $\omega_{0}=1, \beta=0.1, \mu A=1, S_{0}=1, R=1$ and various values of $\alpha$

\begin{tabular}{|c|c|c|c|c|c|c|c|}
\hline$\alpha$ & $E\left[w_{1}^{2}\right]_{\text {exact, } 1}$ & $E\left[w_{1}^{2}\right]_{\mathrm{CL}, 1}$ & Error (\%) & $E\left[w_{1}^{2}\right]_{\mathrm{EM}, 1}$ & Error $(\%)$ & $E\left[w_{1}^{2}\right]_{\mathrm{DM}, 1}$ & Error (\%) \\
\hline 1.0 & 4.4131 & 3.9581 & 10.3102 & 4.2767 & 3.0909 & 4.5614 & 3.3618 \\
\hline 2.0 & 4.0310 & 3.6844 & 8.5978 & 3.9549 & 1.8889 & 4.1930 & 4.0181 \\
\hline 3.0 & 3.6976 & 3.4334 & 7.1448 & 3.6624 & 0.9518 & 3.8610 & 4.4191 \\
\hline 4.0 & 3.4056 & 3.2038 & 5.9244 & 3.3975 & 0.2382 & 3.5629 & 4.6202 \\
\hline 5.0 & 3.1489 & 2.9944 & 4.9075 & 3.1581 & 0.2921 & 3.2960 & 4.6714 \\
\hline 6.0 & 2.9224 & 2.8036 & 4.0656 & 2.9422 & 0.6756 & 3.0573 & 4.6147 \\
\hline 7.0 & 2.7218 & 2.6300 & 3.3715 & 2.7475 & 0.9442 & 2.8438 & 4.4840 \\
\hline 8.0 & 2.5433 & 2.4721 & 2.8008 & 2.5719 & 1.1241 & 2.6528 & 4.3056 \\
\hline 9.0 & 2.3840 & 2.3284 & 2.3324 & 2.4135 & 1.2370 & 2.4817 & 4.0991 \\
\hline 10.0 & 2.2412 & 2.1975 & 1.9480 & 2.2703 & 1.3000 & 2.3281 & 3.8785 \\
\hline
\end{tabular}

dual criterion are remaining about $6 \%$. For large values of $1 / R$, for instance $1 / R=5$, $1 / R=10$, however, the error of the dual criterion method is smallest (about $2 \%$ ).

In Tab. 2 , the parameter $1 / R$ is taken to be 1 , the stiffness parameter $\alpha$ varies from 1.0 to 10.0, other parameters have the same values as in Tab. 1. It is observed that, the dual criterion method gives a good prediction on response errors (about $5 \%$ ) as $\alpha$ varies, and the error of energy method is smallest.

\subsection{The case of two-mode}

Numerical computations used the fixed-point iteration method $[25,26]$ to find approximate mean-square response of the first mode of beam vibration are carried out for 
the nonlinear algebraic system (33) and (43) with unknowns $k_{m, e q}$ in the case of two-mode. The exact solution is obtained from the multiple integral (46) with $M=2$. The obtained numerical results are presented in Tabs. 3 and 4. Tab. 3 shows that, the error of dual criterion method is in good agreement with that of the energy method. In Tab. 4, in general, the dual criterion and energy method yield values that are close to the exact solutions with different value of the stiffness parameter $\alpha$.

Table 3. Mean-square response of $w_{1}$ of the simply supported beam in case of two-mode with $\omega_{0}=1, \alpha=1, \beta=0.1, \mu A=1, S_{0}=1$ and various values of $1 / R$

\begin{tabular}{|c|c|c|c|c|c|c|c|}
\hline $1 / R$ & $E\left[w_{1}^{2}\right]_{\text {exact, } 1}$ & $E\left[w_{1}^{2}\right]_{\mathrm{CL}, 1}$ & Error $(\%)$ & $E\left[w_{1}^{2}\right]_{\mathrm{EM}, 1}$ & Error $(\%)$ & $E\left[w_{1}^{2}\right]_{\mathrm{DM}, 1}$ & Error (\%) \\
\hline 0.01 & 15.6865 & 15.6866 & 0.0007 & 15.6901 & 0.0232 & 15.6921 & 0.0358 \\
\hline 0.02 & 15.6233 & 15.6232 & 0.0006 & 15.6372 & 0.0887 & 15.6449 & 0.1385 \\
\hline 0.05 & 15.2125 & 15.2050 & 0.0494 & 15.2847 & 0.4744 & 15.3290 & 0.7658 \\
\hline 0.10 & 14.0574 & 13.9915 & 0.4688 & 14.2343 & 1.2587 & 14.3672 & 2.2039 \\
\hline 0.20 & 11.4744 & 11.2121 & 2.2857 & 11.6951 & 1.9235 & 11.9358 & 4.0207 \\
\hline 0.50 & 6.7777 & 6.3387 & 6.4772 & 6.8873 & 1.6175 & 7.0652 & 4.2423 \\
\hline 1.00 & 3.9033 & 3.5576 & 8.8571 & 3.9627 & 1.5219 & 4.0385 & 3.4636 \\
\hline 2.00 & 2.0923 & 1.8799 & 10.1519 & 2.1284 & 1.7243 & 2.1529 & 2.8951 \\
\hline 5.00 & 0.8714 & 0.7764 & 10.9030 & 0.8891 & 2.0276 & 0.8936 & 2.5492 \\
\hline 10.00 & 0.4414 & 0.3923 & 11.1312 & 0.4510 & 2.1828 & 0.4522 & 2.4529 \\
\hline
\end{tabular}

Table 4. Mean-square response of $w_{1}$ of the simply supported beam in case of two-mode with $\omega_{0}=1, \beta=0.1, \mu A=1, S_{0}=1, R=1$ and various values of $\alpha$

\begin{tabular}{|c|c|c|c|c|c|c|c|}
\hline$\alpha$ & $E\left[w_{1}^{2}\right]_{\text {exact, } 1}$ & $E\left[w_{1}^{2}\right]_{\mathrm{CL}, 1}$ & Error $(\%)$ & $E\left[w_{1}^{2}\right]_{\mathrm{EM}, 1}$ & Error $(\%)$ & $E\left[w_{1}^{2}\right]_{\mathrm{DM}, 1}$ & Error (\%) \\
\hline 1.0 & 3.9033 & 3.5576 & 8.8571 & 3.9627 & 1.5219 & 4.0385 & 3.4636 \\
\hline 2.0 & 3.5878 & 3.3203 & 7.4557 & 3.6862 & 2.7440 & 3.7299 & 3.9603 \\
\hline 3.0 & 3.3112 & 3.1035 & 6.2725 & 3.4338 & 3.7025 & 3.4526 & 4.2703 \\
\hline 4.0 & 3.0677 & 2.9058 & 5.2784 & 3.2038 & 4.4375 & 3.2038 & 4.4375 \\
\hline 5.0 & 2.8526 & 2.7256 & 4.4511 & 2.9947 & 4.9812 & 2.9808 & 4.4929 \\
\hline 6.0 & 2.6616 & 2.5616 & 3.7582 & 2.8047 & 5.3755 & 2.7807 & 4.4741 \\
\hline 7.0 & 2.4915 & 2.4121 & 3.1849 & 2.6321 & 5.6419 & 2.6010 & 4.3960 \\
\hline 8.0 & 2.3393 & 2.2760 & 2.7071 & 2.4752 & 5.8105 & 2.4394 & 4.2811 \\
\hline 9.0 & 2.2026 & 2.1517 & 2.3090 & 2.3326 & 5.9016 & 2.2938 & 4.1422 \\
\hline 10.0 & 2.0793 & 2.0383 & 1.9738 & 2.2027 & 5.9355 & 2.1623 & 3.9922 \\
\hline
\end{tabular}




\section{CONCLUSIONS}

The method of equivalent linearization is one of effective tools in solving random vibration problems of mechanical systems. In this study, the modal responses of a simply supported beam subjected to a space-wise and time-wise white noise loading are carried out by the dual criterion of equivalent linearization. Our calculations are restricted in two cases of single- and two-mode of beam vibrations. The exact solutions of the original modal equation system obtained by Fokker-Planck equation are available for both cases. A closed form of nonlinear algebraic system is obtained by the dual approach associated with the frequency-response function method for the linearized modal system. In the case of single-mode, the analytical solution of the first mode of the beam is easy to solve explicitly for four methods considered (the exact solution, energy method, conventional linearization and dual criterion method). Also, in the case of two-mode, the closed system is solved by the fixed-point iteration method. Numerical results show that the dual criterion gives a good prediction on the random responses of the beam, especially in the range of strong linearity of system parameters. Further investigations for random vibrations of other beam systems seem to be appropriate in order to verify the advantages of the dual criterion.

\section{ACKNOWLEDGEMENTS}

This research is funded by Vietnam National Foundation for Science and Technology Development (NAFOSTED) under grant number: "107.04-2015.36".

\section{REFERENCES}

[1] R. C. Booton. Nonlinear control systems with random inputs. Circuit Theory, IRE Transactions on, 1, (1), (1954), pp. 9-18.

[2] I. E. Kazakov. An approximate method for the statistical investigation of nonlinear systems. Trudy VVIA im Prof. NE Zhukovskogo, 394, (1954), pp. 1-52. (in Russian).

[3] T. K. Caughey. Equivalent linearization techniques. The Journal of the Acoustical Society of America, 35, (11), (1963), pp. 1706-1711.

[4] T. K. Caughey. Nonlinear theory of random vibrations. Advances in Applied Mechanics, 11, (1971), pp. 209-253.

[5] L. Socha. Linearization methods for stochastic dynamic systems, Vol. 730. Springer Science \& Business Media, (2007).

[6] T. S. Atalik and S. Utku. Stochastic linearization of multi-degree-of-freedom non-linear systems. Earthquake Engineering \& Structural Dynamics, 4, (4), (1976), pp. 411-420.

[7] E. T. Foster. Semilinear random vibrations in discrete systems. Journal of Applied Mechanics, 35, (3), (1968), pp. 560-564.

[8] W. D. Iwan and I.-M. Yang. Application of statistical linearization techniques to nonlinear multidegree-of-freedom systems. Journal of Applied Mechanics, 39, (2), (1972), pp. 545-550.

[9] R. C. Micaletti, A. Ş. Çakmak, S. R. K. Nielsen, and H. U. Köylüolu. Error analysis of statistical linearization with Gaussian closure for large-degree-of-freedom systems. Probabilistic Engineering Mechanics, 13, (2), (1998), pp. 77-84.

[10] I. Elishakoff, L. Andriamasy, and M. Dolley. Application and extension of the stochastic linearization by Anh and Di Paola. Acta Mechanica, 204, (1-2), (2009), pp. 89-98. 
[11] N. D. Anh and L. X. Hung. An improved criterion of Gaussian equivalent linearization for analysis of non-linear stochastic systems. Journal of Sound and Vibration, 268, (1), (2003), pp. 177-200.

[12] L. Socha. Linearization in analysis of nonlinear stochastic systems. Applied Mechanics Reviews, 44, (1991), pp. 99-422.

[13] L. Socha. Linearization in analysis of nonlinear stochastic systems: recent results-part I: Theory. Applied Mechanics Reviews, 58, (3), (2005), pp. 178-205.

[14] L. Socha. Linearization in analysis of nonlinear stochastic systems, recent results-part II: Applications. Applied Mechanics Reviews, 58, (5), (2005), pp. 303-315.

[15] S. H. Crandall. A half-century of stochastic equivalent linearization. Structural Control and Health Monitoring, 13, (1), (2006), pp. 27-40.

[16] I. Elishakoff and L. Andriamasy. The tale of stochastic linearization technique: Over half a century of progress. Springer, (2012).

[17] S. H. Crandall and W. D. Mark. Random vibration in mechanical systems. Academic Press, (2014).

[18] Y.-K. Lin. Probabilistic theory of structural dynamics. New York, McGraw-Hill, (1973).

[19] J. B. Roberts and P. D. Spanos. Random vibration and statistical linearization. New York, Wiley, (1990).

[20] R. E. Herbert. Random vibrations of a nonlinear elastic beam. The Journal of the Acoustical Society of America, 36, (11), (1964), pp. 2090-2094.

[21] R. E. Herbert. On the stresses in a nonlinear beam subject to random excitation. International Journal of Solids and Structures, 1, (2), (1965), pp. 235-242.

[22] P. Seide. Nonlinear stresses and deflections of beams subjected to random time dependent uniform pressure. Journal of Engineering for Industry, 98, (3), (1976), pp. 1014-1020.

[23] W. D. Iwan and R. G. Whirley. Nonstationary equivalent linearization of nonlinear continuous systems. Probabilistic Engineering Mechanics, 8, (3), (1993), pp. 273-280.

[24] I. Elishakoff, J. Fang, and R. Caimi. Random vibration of a nonlinearly deformed beam by a new stochastic linearization technique. International Journal of Solids and Structures, 32, (11), (1995), pp. 1571-1584.

[25] J. Fang, I. Elishakoff, and R. Caimi. Nonlinear response of a beam under stationary random excitation by improved stochastic linearization method. Applied mathematical modelling, 19, (2), (1995), pp. 106-111.

[26] N. D. Anh, I. Elishakoff, and N. N. Hieu. Extension of the regulated stochastic linearization to beam vibrations. Probabilistic Engineering Mechanics, 35, (2014), pp. 2-10.

[27] N. D. Anh, N. N. Hieu, and N. N. Linh. A dual criterion of equivalent linearization method for nonlinear systems subjected to random excitation. Acta Mechanica, 223, (3), (2012), pp. 645-654.

[28] N. D. Anh, V. L. Zakovorotny, N. N. Hieu, and D. V. Diep. A dual criterion of stochastic linearization method for multi-degree-of-freedom systems subjected to random excitation. Acta Mechanica, 223, (12), (2012), pp. 2667-2684.

[29] S. H. Crandall and A. Yildiz. Random vibration of beams. Journal of Applied Mechanics, 29, (2), (1962), pp. 267-275.

[30] D. Middleton. An introduction to statistical communication theory. McGraw-Hill New York, (1960). 